\title{
Kaposi Sarcoma with Abscessing Clinicopathologic Morphology
}

\author{
Naidoo $\mathrm{N}^{1}$, Ramdial $\mathrm{PK}^{2 *}$, Kuppusamy $\mathrm{JB}^{2}$, Naidoo $\mathrm{T}^{2}$ and Pillay $\mathrm{B}^{3}$
}

${ }^{1}$ Department of Surgery, Port Shepstone Regional Hospital and Department of General Surgery, School of Clinical Medicine, University of KwaZulu-Natal, Durban, KwaZulu-Natal, South Africa

${ }^{2}$ Department of Anatomical Pathology, National Health Laboratory Service, University of KwaZulu-Natal, Durban, KwaZulu-Natal, South Africa

${ }^{3}$ Department of Vascular/Endovascular Surgery, Nelson R Mandela School of Medicine, University of KwaZulu-Natal, Inkosi Albert Luthuli Central Hospital, Durban, KwaZulu-Natal, South Africa

\section{Abstract}

Background: The AIDS epidemic heralded an expanded spectrum of Kaposi sarcoma (KS) variants but to date $\mathrm{KS}$ masquerading as an abscess on clinical and histopathological assessment is undocumented.

Methods: This 3 year retrospective study re-appraised the clinicopathological profile of all biopsies of KS that presented as abscesses clinically and demonstrated abscessing or suppurative microscopic features.

Results: Ten males and 9 females form the study cohort. The clinical diagnosis in 11 patients was exclusively that of an abscess. Tuberculosis and abscess were clinical differential diagnoses in 8 patients. The average CD4 count was $261 \mathrm{cells} / \mathrm{mm}^{3}$. Abscessing KS was a sentinel of HIV infection and AIDS in 4 patients. Of 11 patients on HAART, 3 were virally suppressed. The pathology appeared within 3 months of HAART commencement in 5 patients; repeat CD4 counts and viral load assessments were not available for scrutiny. Microscopically, 17/19 initial biopsies with abscessing morphology demonstrated typical features of KS at least focally, while $2 / 19$ had an exclusive abscess-like morphology. Spindle cell heterogeneity was present with co-existence in all biopsies of myofibroblasts and malignant spindle cells, the latter were HHV8-LANA-1 positive. Special stains and polymerase chain reaction investigation for infections were negative and were crucial in excluding an infective cause. Sequestrum was noted focally in the exudate of one biopsy but the patient's demise precluded assessment of underlying osteitis. Folliculitis was not present.

Conclusion: HHV8-LANA-1 immunostaining underpins the identification of KS that masquerades as an abscess clinically and may mimic a spectrum of infections microscopically. Heightened awareness of this inflammatory response is pivotal to its diagnosis. The exact cause of the abscessing morphology was unconfirmed in the present study, but on-going investigation is critical to determine the pathogenesis of this, hitherto undescribed, clinicopathological profile of KS, that may predicate altered therapeutic approaches.

Keywords: Kaposi sarcoma; Suppuration; Pyogenic; Abscess; HIV; AIDS

\section{Introduction}

Kaposi sarcoma (KS), a multicentric angioproliferative condition [1], is associated with Human Herpes Virus 8 (HHV8) infection and widespread anatomical involvement, including cutaneous, mucosal and internal visceral sites. Described originally by Moritz Kaposi over a century ago [2], it is categorized into 4 clinico-epidemiological forms: classic, African (endemic), AIDS-associated (epidemic) and iatrogenic KS subtypes. KS is regarded as a low grade tumor but an aggressive course occurs in $>50 \%$ of patients in the AIDS context [3]. Irrespective of the clinical subgroup of KS, similar cutaneous histopathological features occur for well-known KS variants that encompass 3 distinctive morphological patch, plaque and nodular stages with potential for morphological overlap. The HIV/AIDS era, however, has introduced an expanded spectrum of histomorphological variants [4-6]. Furthermore, the microscopic features of KS lesions are impacted by co-morbid, concomitant immunosuppressive diseases, and anti-retroviral druginduced flares and regression [2,7-11]. Hence, the histomorphological spectrum of KS is currently characterized by well-known established, uncommon and emerging variants [12]. Notwithstanding these emerging variants, to date, clinically unsuspected KS lesions masquerading as abscesses, with histopathological features of KS masked by exuberant suppurative inflammation, justifying an appellation of 'abscessing' Kaposi sarcoma, is a hitherto unreported phenomenon, with potential serious patient management implications.

This study was therefore undertaken to assess the clinicopathological features of KS with abscessing morphology and to highlight potential diagnostic mimicry and pitfalls. In addition, potential pathogenetic mechanisms for the abscessing presentation are discussed.

\section{Materials and Methods}

This is a descriptive 3 year retrospective study that involved a reappraisal of all biopsies of KS with an abscessing morphology that were diagnosed in the Department of Anatomical Pathology, National Health Laboratory Service, Durban, KwaZulu-Natal from 1 January 2012 to 31 December 2014. The SNOMED word search engine targeting 'Kaposi' and 'abscess' was employed to identify confirmed biopsies of KS with abscessing morphology for study purposes. The clinical details that were obtained from the histopathology request forms included age, sex, race, presenting symptoms, laterality, imaging findings, HIV status, CD4 counts, HIV viral load, clinical differential diagnosis, exposure to highly active anti-retroviral therapy (HAART) and the interval between HAART initiation and

*Corresponding author: PK Ramdial, Department of Anatomical Pathology, Leve 3, Laboratory Building, Inkosi Albert Luthuli Central Hospital, 800 Vusi Mzimela Road, Mayville, 4058, KwaZulu-Natal, South Africa; Tel: +27 (0)31 2402693; Fax: + 27 (0)31 2402610; E-mail: ramdial@ukzn.ac.za; ramdialpk@gmail.com

Received August 14, 2017; Accepted September 02, 2017; Published September 09,2017

Citation: Naidoo N, Ramdial PK, Kuppusamy JB, Naidoo T, Pillay B (2017) Kaposi Sarcoma with Abscessing Clinicopathologic Morphology. J AIDS Clin Res 8: 726. doi: 10.4172/2155-6113.1000726

Copyright: @ 2017 Naidoo N, et al. This is an open-access article distributed under the terms of the Creative Commons Attribution License, which permits unrestricted use, distribution, and reproduction in any medium, provided the original author and source are credited. 
disease onset. The histopathology report provided information on gross characteristics of the submitted specimen.

Using the histopathology accession numbers, slides were retrieved from the archive of the Department of Anatomical Pathology/National Health Laboratory Service, Durban, KwaZulu-Natal for re-evaluation. Sections of biopsies that had faded were recut and stained with hematoxylin and eosin to assess the presence of chronic suppurative inflammation typical of abscess wall, morphologic subtype of KS, ulceration, sequestrum and bacterial colonies. Ziehl Neelsen, periodic acid Schiff, Warthin Starry and Brown Brenn Hopps stains were undertaken to identify potential intra-lesional mycobacteria, fungi, Bartonella species and Gram-positive and Gram-negative bacteria, respectively. Immunohistochemical immunoprofiling for HHV8-LANA-1 (Novocastra, Newcastle-UponTyne , UK, clone13B10, 1:40 dilution) and endothelial cells using antiCD34 antibody (DakoCytomation, Glostrup, Denmark, clone QBEND-10, 1:40 dilution) and anti-CD31 antibody (DakoCytomation, Glostrup, Denmark, clone JC70, 1:30 dilution) were undertaken on $2 \mu \mathrm{m}$ thick sections cut from archival wax blocks. The tissue sections were subjected to heat-assisted microwave antigen retrieval and manual polymer-based immunohistochemical staining with diaminobenzidine as the chromogen, using a standard immunohistochemical protocol with positive and negative controls. Genomic DNA was extracted from formalin-fixed paraffin-embedded tissues using the QIAamp DNA formalin- fixed tissue (Qiagen, Hilden, Germany) according to the manufacturer's protocol. Quantified DNA quality was thereafter assessed by PCR amplification of the 159 bp product of the reference gene, $\beta$-actin [13]. PCR investigations were undertaken to detect Mycobacterium tuberculosis (MTb) and Bartonella species. Samples that were negative following agarose gel electrophoresis on the first outer PCR were then subjected to nested PCR. All PCR amplification was performed on a CFX96 Realtime PCR system (BIO-RAD, CA, USA). Bartonella species identification was accomplished by the double PCR of a 126-326 bp region of the internal transcribed spacer region of ribosomal RNA using the primers (16SrDNA F: CGTTTCTCTTTCTTCAGATGATG and 16SrDNA R: CTCTAACCAACTGAGCTACAAG). MTb complex identification was accomplished by PCR targeting of a 220 bp segment of IS6110 using the primers O1: CGGGACCACCCGCGGCAAAGCCCGCAGGAC; O2: CATCGTGGAAGCGACCCGCCAGCCCAGGAT) and a $123 \mathrm{bp}$ segment using inner primers (I1: CCTGCGAGCGTAGGCGTCGG and I2: CTCGTCCAGCGCCGCTTCGG).

Permission to conduct the study was obtained from the University of KwaZulu-Natal Biomedical Research Ethics Committee (BE366/15).

\section{Results}

5813 biopsies of KS were confirmed in the study period. Of these, 28
(0.44\%) patients with biopsies of KS with abscessing morphology were identified. Adequate clinical information of 19 patients was available; biopsies from these 19 patients form the study cohort.

\section{Clinical details}

There were 10 males and 9 females. The average patient age was 27.8 (range: 12-50) years. The patients presented with painful, expanding masses in the toe (10), thigh (4), trunk (1), arm (1), palate (1), neck (1) and anus (1). The mass lesions were recognized as abscesses clinically and the walls thereof were biopsied during the incision and drainage procedure. The average CD4 count was 261 (range: 7-1002) cells/ $\mathrm{mm}^{3}$. Abscessing KS was a sentinel of the HIV infection in patients 4, 10, 12 and 14 (Table 1). Patients $1,6,8,12$ and 18 were on antituberculous therapy for pulmonary tuberculosis. Of 11 patients on HAART, viral immunosuppression was confirmed in 3 (patients 1, 5, 11). The pathology appeared within 2 and 3 months of commencement of HAART in 3 and 2 patients, respectively. Patient 10 had recurrence of KS after chemoradiation. Of 4 patients (patients 1,6,12,18) with lymphadenopathy, 2 had Castleman disease, confirmed in cervical and inguinal lymph node biopsies. The other 2 demonstrated reactive lymphoid hyperplasia and sinus histiocytosis.

The clinical diagnosis was exclusively that of an abscess in 11 patients. Tuberculosis and abscess were clinical differential diagnoses in 8 patients. KS was not a clinical consideration for any of the abscessing lesions, although disseminated cutaneous KS was noted in all patients and pleural and oral lesions in 1 and 4 patients each, respectively, on follow-up assessment.

\section{Pathological findings}

Seventeen patients had a single biopsy while 2 patients had 2 biopsies each. A total of 21 biopsies were reviewed for study purposes.

\section{Gross details}

All biopsies were of incisional wedge type and were noted to be hemorrhagic with cream and yellow necrotic foci, compatible with the clinical impression of granulating abscess wall and associated abscess contents.

\section{Microscopic details}

Of the 19 initial biopsies with abscessing morphology, 17 demonstrated typical features of KS at least focally while $2 / 19$ (patients $5,15)$ had an exclusive abscess-like morphology. The focal areas of KS in 17/19 biopsies demonstrated a solid arrangement of hyperchromatic spindle cells with intervening slit-like vascular channels (Figure 1A), hyaline globules, hemosiderin pigment, variable mitoses and HHV8-

\begin{tabular}{|c|c|c|c|c|c|c|c|c|c|}
\hline P/No. & Age/Sex & Site & HAART & CD4 & P/No. & Age/Sex & Site & HAART & CD4 \\
\hline 1. & 28/female & Toe & Yes & 512 & 11. & $27 /$ male & Palate & Yes & 629 \\
\hline 2. & $21 /$ male & Thigh & Yes & 421 & 12. & 23/female & Neck & No & 130 \\
\hline 3. & 42/male & Toe & Yes & 285 & 13. & 21/male & Ileum & Yes & 129 \\
\hline 4. & $37 /$ male & Toe & NK & 7 & 14. & 29/female & Thigh & No & 85 \\
\hline 5. & 50/female & Arm & Yes & 384 & 15. & 33/female & Thigh & No & 212 \\
\hline 6. & $21 /$ male & Toe & Yes & 250 & 16. & 21/female & Toe & Yes & 286 \\
\hline 7. & 12/female & Trunk & Yes & 1002 & 17. & $35 /$ male & Toe & Yes & 108 \\
\hline 8. & 25/male & Toe & NK & 283 & 18. & 22/female & Toe & NK & 41 \\
\hline 9. & 29/male & Toe & Yes & 51 & 19. & 21/female & Toe & NK & 131 \\
\hline 10. & $31 /$ male & Thigh & No & 13 & & & & & \\
\hline
\end{tabular}

Key: P/No.: Patient Number; CD4: CD4 Count in cells/mm³ HAART: Exposure to Highly Active Anti-Retroviral Therapy; NK: Not Known

Table 1: Summary of clinicopathological details. 


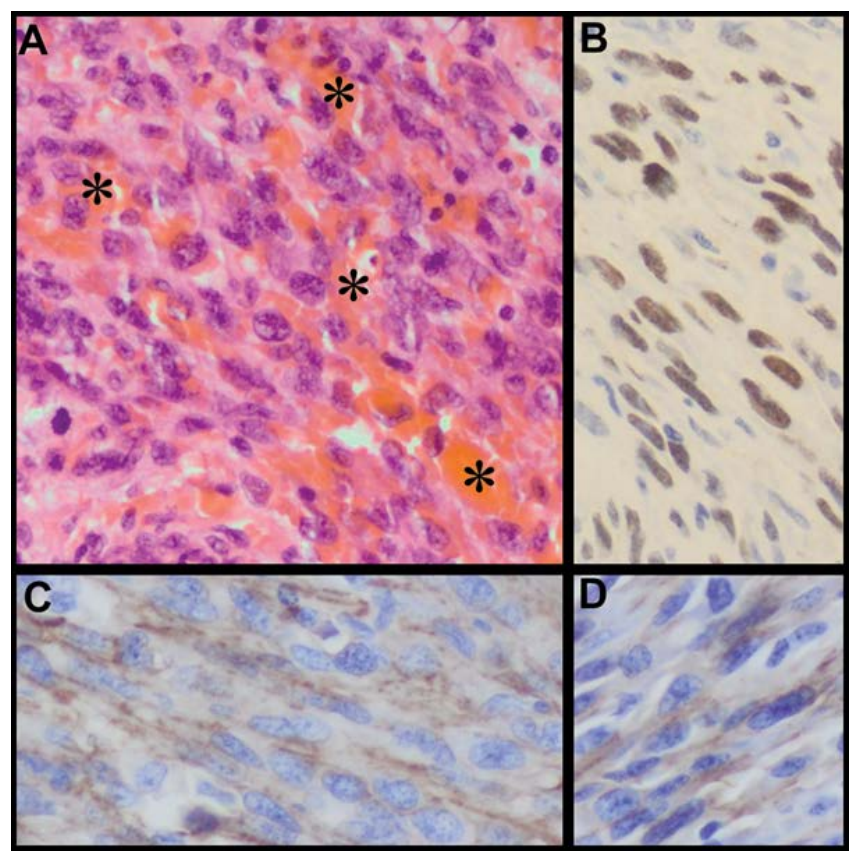

Figure 1: Nodular Kaposi sarcoma (A) composed of hyperchromatic spindle cells and intervening slit-like vascular channels (asterisks) (H\&E, 240X) demonstrating brown nuclear HHV8-LANA-1 (B) (480X) and brown cytoplasmic CD34 (C) (480X)and CD31 (D) (480X) immunopositivity.

LANA-1 (Figure 1B), CD34 (Figure 1C) and CD31 (Figure 1D) immunopositivity. The abscessing morphology that dominated these 17 biopsies, noted centrally or in the deep, subcutaneous aspect of the sample, demonstrated organizing, chronic suppurative inflammation in granulation tissue, typical of an abscess wall and contents (Figures $2 \mathrm{~A}$ and $2 \mathrm{~B}$ ). However, the spindle cells within the granulation tissue were heterogeneous; some were normochromatic and resembled myofibroblasts while others were hyperchromatic (Figure 2C). This prompted the conduction of HHV8-LANA-1 and $\alpha$-smooth muscle actin immunostaining on these biopsies, that confirmed the divergent spindle cell population (Figures $2 \mathrm{D}$ and $2 \mathrm{E}$ ), composed of a dual population of malignant KS spindle cells (Figure 2D) and myofibroblasts in the admixed organizing granulation tissue response (Figure 2E). Second deeper and wider biopsies from the same tumor site in 2 patients that had exclusive abscess-like morphology in the initial biopsy confirmed typical HHV8-LANA-1 positive KS.

The sarcomatous component from patients $4,10,12,13$ and 18 who had HAART exposure for 2 to 3 months, also demonstrated an infiltrate of lymphoid cells, neutrophils and histiocytes (Figures 3A and 3B). Eight biopsies demonstrated variable epidermal ulceration and nonspecific, superficial bacterial colonization of the inflammatory exudate, but there was no connection between the ulcerated component and the deeper abscessing morphology (Figures 3C and 3D). Tuberculosis and bacillary angiomatosis were excluded in all biopsies by the lack of organisms on special infective stains. In addition PCR investigation for tuberculosis and bacillary angiomatosis were negative in all samples (Figures 4A and 4D). Focal sequestrum was identified within the abscessing component (patient 4) but attempts to confirm connection of the abscessing tumor with underlying osteitis on clinical review and imaging studies failed because the patient died shortly after biopsy. Folliculitis was not present in any biopsy.

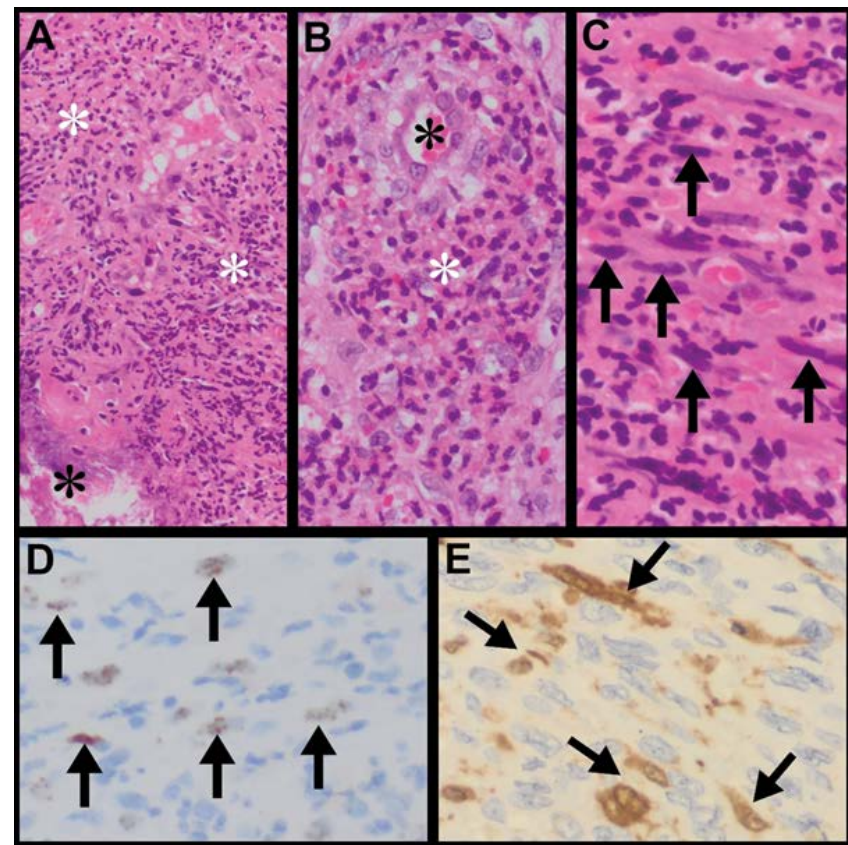

Figure 2: Abscessing morphology with (A) chronic suppurative inflammation in granulation tissue (white asterisks) and central breakdown with necrotic and karyorrhectic debris (black asterisk) (H\&E, 120X). High power demonstration (B) of vasculature (black asterisk) in granulation tissue and suppurative inflammation (white asterisk) (H\&E, 240X). High power demonstration of hyperchromatic spindle cells (C, arrows) (H\&E, 240X), HHV8-LANA-1 positive spindle cells (D, arrows) (480X) and a-smooth muscle actin immunopositive myofibroblasts in granulation tissue (E, arrows) (480X).

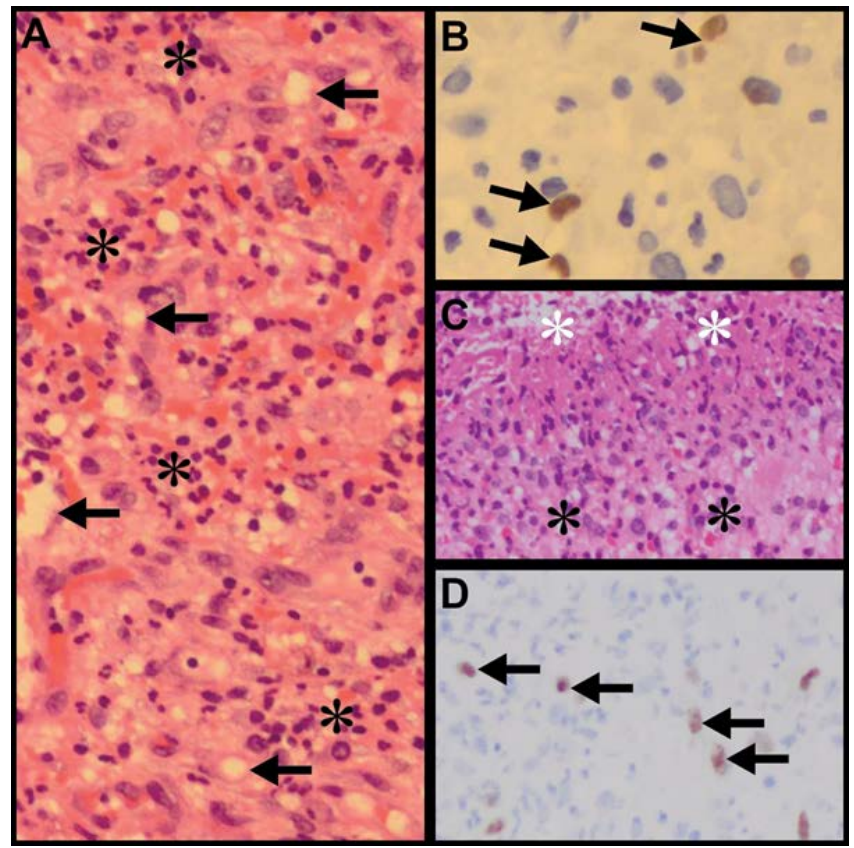

Figure 3: Biopsy from patient 9 in third month of anti-retroviral therapy demonstrating inflamed granulation tissue with capillaries (A, arrows) and neutrophils ( $A$, asterisks) (H\&E, 120X) and HHV8-LANA-1 positive cells therein ( $B$, arrows). Acute inflammatory exudate ( $C$, white asterisks) on the superficial aspect of ulcerated Kaposi sarcoma (C, black asterisks) with scattered HHV8LANA-1 positive spindle cells ( $D$, arrows). 


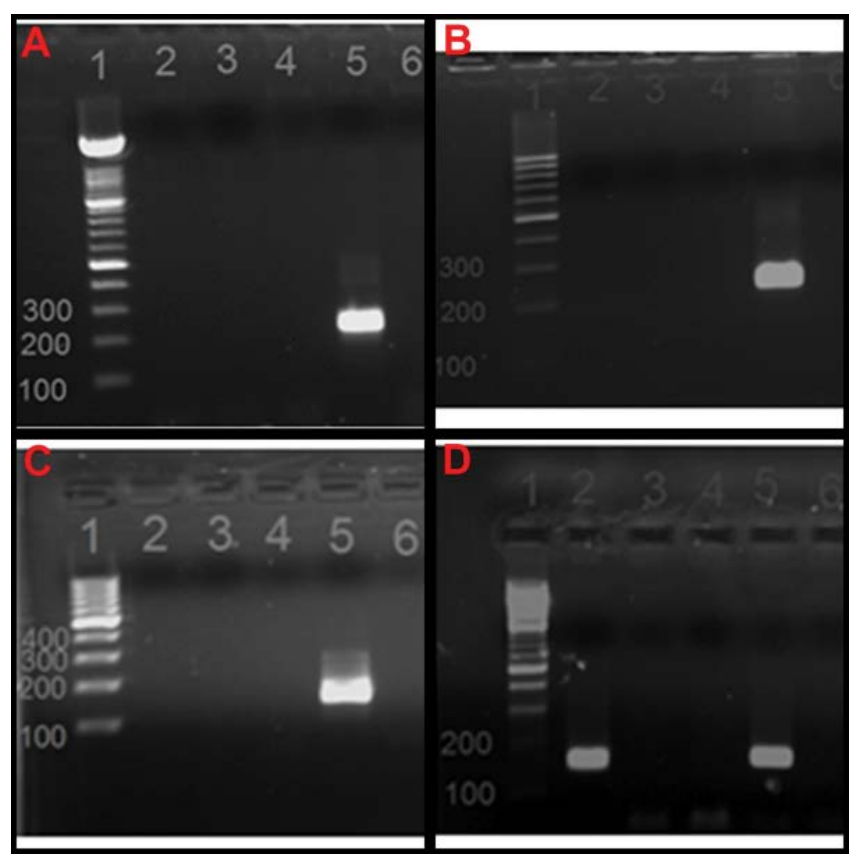

Figure 4: Agarose gel electrophoresis results in tissue from biopsy of Patien 6: lane 2 in $A, B$ and $C$ demonstrating negative $P C R$ result for $M$. tuberculosis (IS6110, outer (A)) and (IS6110, inner/nested (B)) and Bartonella species (C) in patient samples. Confirmed external positive PCR controls in lane 5 of $A, B$ and $\mathrm{C}$ for same markers. Reference gene, $\beta$-actin, positive (D) confirming presence of amplifiable extracted DNA in patient sample (lane2) and in external positive control (lane 5).

Key for A-D: lane 1: molecular weight marker; lane 2: patient sample; lane 3 tissue sectioning control to measure cross-contamination during sectioning; lane 4: extraction control to measure cross-contamination during extraction procedure; lane 5: external positive control; lane 6: non-template control)

\section{Discussion}

Although first diagnosed in $1872, \mathrm{KS}$ remained a rare neoplasm until the late 1980s when the AIDS epidemic caused its increased incidence and launched it as the most common AIDS-defining malignancy [2]. In the pre-HIV/AIDS era, the incidence of KS in Europe and North America ranged from 0.01 to 0.06 per 100,000, although an increased incidence was noted in patients post-renal transplantation and on immunosuppression $[1,2]$. In a transplant setting, a patient is 400 500 times more likely to develop KS within 3 to 124 months, than in the general population [14]. While classic KS is typified as a global indolent disease with a predilection for elderly Mediterranean men, African (endemic) KS affects young Black men predominantly but also manifests with lymphadenopathy in childhood [1]. Iatrogenic KS follows immunosuppressive therapy post-organ transplantation, long term corticosteroids usage and chemotherapy administration while epidemic KS is associated with AIDS [3]. Historically, KS has been associated with clinical and histopathological heterogeneity, although the latter is not unique to any specific clinical disease stage or presentation. The emergence of new KS variants in the AIDS era has not only expanded the histopathological spectrum but also introduced a plethora of mimickers and diagnostic pitfalls in its wake $[12,15]$.

Currently, the histopathological diagnostic challenges include those related to mimicry of common well-recognized, uncommon poorly-recognized and newly-recognized KS variants, procedure and treatment-related changes and the impact of co-morbid co-lesional pathology on KS lesions [16]. Apart from heightened awareness and recognition of the changing face of KS in the AIDS era, diagnostic confirmation has also been facilitated by the relative recent discovery of Human herpesvirus-8 (HHV8), also labelled KS-associated herpes virus, as the viral etiopathogenesis of KS [17]. This has facilitated improved understanding of $\mathrm{KS}$ and therapeutic possibilities and distinction from diagnostic masqueraders [17-23]. The commercial availability of HHV-8 LANA-1 immunohistochemical antibodies and HHV8 PCR studies has enabled the diagnostic confirmation of KS in challenging histodiagnostic settings [19-22]. HHV-8 LANA-1 is a nuclear immunohistochemical antigen that is expressed by all cells that are latently infected by the virus. It is more reliable than PCR investigation as it demonstrates the nuclear immunolocalization of the viral product, in contrast to potential HHV-8 passenger lymphocytes and non-specific amplification, that may produce false positive results on PCR testing [20,21].

Because of the clinical diagnosis and the presence of granulation tissue with a neutrophil-rich component, abscess is a compelling microscopic diagnosis [24] and risky pitfall. However, in all biopsies, careful assessment revealed the presence of short spindle cell fascicles at the periphery of the suppurative component or admixed hyperchromatic spindle cells, which is not a usual feature of an abscess wall. Abscess wall histomorphology mainly that of inflamed granulation tissue is characterized by a random arrangement of myofibroblasts and vasculature. Immunophenotypically, the spindle cell component in abscess wall and in granulation tissue is of myofibroblastic lineage and will stain with myoid markers, including a-smooth muscle actin, muscle specific actin, calponin and variably with desmin [25]. In contrast, the spindle cells in KS lack a myoid immunophenotype, stain negatively with myoid immunomarkers but positively with the endothelial markers, CD31 and CD34; these endothelial markers do not stain myofibroblasts in granulation tissue [26]. Granulation tissue may assume a pseudosarcomatous histomorphology and mimic KS closely $[25,26]$. In this context, negative HHV8 immunostaining will distinguish KS from abscess wall.

In the context of AIDS, tuberculous and fungal infections are potential mimickers of KS with an abscessing morphology. Whilst the classic granulomatous response of tuberculosis sets it apart from KS, the anergic response in immunosuppressed patients lacks a granulomatous phenotype. The anergic tuberculous responses may be lymphohistiocytic, acellular or neutrophil-rich with abundant karyorrhexis [27-30]. Although documented more than 6 decades ago [31], the neutrophilic and karyorrhectic form of anergic TB has received more attention in the HIV and AIDS era. Granuloma-poor, karyorrhectic, mycobacteriarich anergic responses have been associated with low CD4 counts and associated impaired cell-mediated and humoral immunity [27,28]. Heightened awareness of the anergic tuberculous responses underpin the conduction of mycobacterial stains for the identification of myriad acid fast bacilli, typical of this tuberculous response. However, a negative infective profile should not default to a diagnosis of abscess because of the acute inflammatory background. Heightened recognition of the abscessing morphology of KS also underpins the conduction of HHV8 stains, pivotal to KS diagnosis.

Bacillary angiomatosis, caused by Bartonella sp., is a known clinical and histopathological mimicker of KS because of the clinical morphology and microscopic vessel-rich morphology [12,16]. Bacillary angiomatosis is typically neutrophil-rich; in such settings, abscessing KS may be misdiagnosed as bacillary angiomatosis, especially as both lesions stain positively with endothelial markers. However, HHV8-LANA-1 is negative in bacillary angiomatosis $[12,16,21]$. Hence, the gold standard in distinguishing abscessing KS from its mimickers is the heightened 
clinicopathological awareness of an abscessing KS presentation and the conduction of HHV8-LANA-1 immunohistochemistry on biopsied tissue sections for diagnostic confirmation.

The exact pathogenesis of abscessing KS remains enigmatic and speculative, but the hypothetical pathogenetic possibilities highlight, not only potential patient prognostication and management implications, but also foreground a new inflammatory paradigm of KS, that requires accelerated investigation for improved understanding. The possible pathogenetic mechanisms for the intra-tumoral neutrophilic influx are categorized into those related to local, systemic HAART-related [32-35] and systemic HAART-unrelated [36,37] mechanisms (Table 2), the latter based on responses caused by tumor-associated neutrophils (TANs) in a wide range of cancers [38-50]. In this study, the local mechanisms for intra-tumoral suppuration include origin of the suppurative component from acute folliculitis in hair-bearing skin, post-traumatic and foreign body reactions, entrapment of superficial acute inflammatory exudate and underlying osteitis. In the present study, only lesional tissue was submitted for appraisal. Careful appraisal of larger excision samples, including of the adjacent skin, will enable assessment of folliculitis in paralesional skin. A toe biopsy contained a splinter, albeit away from the suppurative component. The role of such traumatic insults and foreign bodies in the pathogenesis of the abscessing presentation requires ongoing appraisal. Although not confirmed in the present study, underlying osteitis, especially in destroyed toes, may be the origin of suppuration. Imaging studies and the identification of sequestrum in such biopsies will facilitate such a hypothesis. Finally, many biopsies were ulcerated. The abscessing component was not continuous with the surface, but deep dermal extension of superficial inflammatory exudate and subsequent entrapment thereof, following cicatrisation of the surface connection is a plausible mechanism, especially in older lesions. Wedge biopsies were assessed in this study. Complete appraisal of excised 'abscesses' may be more helpful in confirming the evolution of such entrapment in serial tissue sections.
HAART-associated immune reconstitution inflammatory syndrome (IRIS), also known as immune restoration disease, may be characterized by lesional neutrophilic influx [51-54]. Described in HIV-infected patients for the first time in 1992, IRIS represents an inflammatory host response to a spectrum of antigens in individuals with rapid immune recovery. Classified into unmasking and paradoxical IRIS presentations, with a temporal association with HAART use, increase in CD4 counts and decrease in viral load, the former refers to the mounting of a host response to undetected immunosuppression-related infections during immune recovery. In contrast, paradoxical IRIS occurs despite effective antimicrobial therapy because of infection deterioration caused by a host immune response to antigenic products of degenerating microbes [51]. Occurring in a wide range of infective and non-infective diseases [51-56], IRIS is documented uncommonly in KS [4,56-59]. Major and minor diagnostic criteria have been documented for KS-associated IRIS. The former include abrupt clinical deterioration of pre-existing KS (paradoxical KS-IRIS) or a new presentation of prior unrecognized KS (unmasking KS-IRIS) following initiation or re-initiation of HAART or use of an altered more active therapeutic regime [33,57]. These reactions occur with a simultaneous decrease of at least $1 \log 10$ of the HIV RNA serum levels during the IRIS manifestation or 2 of 3 minor criteria. The latter encompass a 2-fold rise in the CD4+ Tcell level post HAART exposure, an increase in the HHV8 antibody response and spontaneous resolution of KS in the absence of specific chemotherapy but with continued HAART exposure [33].

The pathogenetic basis of KS-associated IRIS is an exaggerated immune response to HHV8, the infective etiological agent of KS. Whilst HAART does not target HHV8, it acts on HIV, decreasing the viral load and improving host immunity that then mounts a new or heightened reaction to HHV8 [35]. Notwithstanding the defined clinical diagnostic criteria for KS-associated IRIS, the histomorphological profile thereof, especially the phenotypic and immunophenotypic spectrum of the cellular inflammatory component in IRIS lesions, is poorly

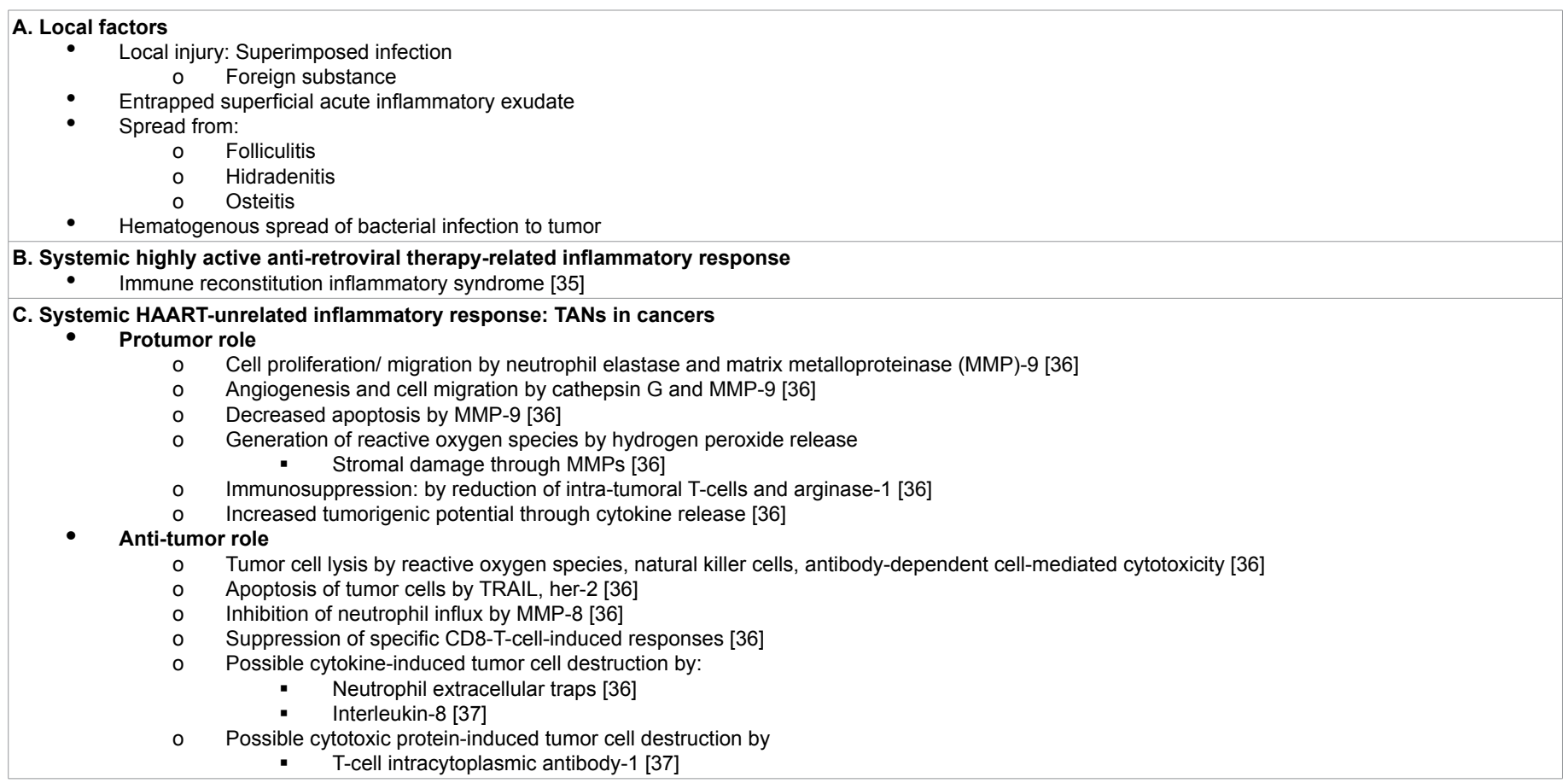

Key: TANs: Tumor-Associated Neutrophils; TRAIL: Tumor-Necrosis Factor Related Apoptosis-Inducing Ligand

Table 2: Potential pathogenetic mechanisms of 'abscessing' Kaposi sarcoma. 
documented. The histomorphological features that have been briefly described include either that of the typical spindle cell reaction of KS or of an intra-tumoral mononuclear inflammatory cell infiltrate, especially of CD3-positive T-lymphocytes [8,34,56,57]. While a neutrophil-rich immune response is described in cryptococal and tuberculous IRIS reactions, it is not documented in KS, to date [53,54]. In the present study, 5 patients manifested with expanding lesions within 2 to 3 months of initiation of HAART. While it is tempting to implicate an immune reconstitution basis for the abscessing morphology in these patients because of the temporal association with HAART exposure, follow-up CD4 counts and viral load assays, pivotal to the diagnosis of IRIS [33], were not traceable, to formalize a definitive hypothesis but require investigation in future studies.

TANs, initially regarded as bystander cells, are now a well-recognized component of the tumor milieu [36], and require consideration in KS, especially when local factors and KS-associated IRIS are not feasible pathogenetic options. The cytokines and chemokines in neutrophil granules may recruit additional cells and are capable of effecting protumoral and anti-tumoral activity (Table 2). TANs have been identified in hematological (Hodgkin and non-Hodgkin anaplastic lymphomas [37,38], plasmacytoma [39], histiocytic sarcoma [40]), epithelial (esophageal [41], gastric [42], pancreaticobiliary [43,44], lung [45], head and neck squamous [36], breast [46,50], renal [47], urinary bladder carcinomas [48]) and germ cell [49] malignancies. In some settings, the neutrophilic influx into tumors has been described as 'pyogenic' [37], 'suppurative' [60] or an 'abscess' [38$40,46]$ in clinical and histopathological presentations. Malignant tumors involving the lung [45], face [39] and bone [38] have masqueraded as an abscess clinically but to the best of our knowledge, KS masquerading as an abscess clinically has been documented only once to date, in a vulval location [61].

This study stands apart as the 'abscessing' KS clinical presentation was also recapitulated on microscopic assessment. KS is of endothelial origin and endothelial cells are pivotal in leucocyte recruitment and regulation thereof [62] through endothelial expression of selectin receptors and synthesis of chemokines and other chemotactic agents. It is therefore surprising that TANs are not part of the protypical inflammatory response of KS [32]. Abrogation of leucocyte recruitment by lysosomal degradation of endothelial ICAM-1 and CD31 by HHV-8 has been hypothesized [63]. More recently, inhibition of neutrophilic migration by HHV8-infected endothelial cells through an interleukin- 6 mechanism has also been proposed. Hence, while KS is apparently equipped with unique mechanisms that preclude the intra-tumoral localization of neutrophils [62], the present study demonstrates breakdown of this property by virtue of the dense neutrophilic accumulation. Future investigation of the pathogenesis of KS with a clinicopathologic 'abscessing' morphology is mandatory to unravel the pathogenesis and prognostic role of this hitherto undocumented presentation, and to investigate potential innovative treatment tools.

\section{Conclusion}

KS with a neutrophil-rich infiltrate dense enough to assume clinical abscessing morphology is unrecognized to date, and even in high AIDS incidence regions such as ours, remains relatively rare. It is uncertain whether this is a truly rare presentation of KS or an under-recognized entity. Notwithstanding this, optimal appraisal of all abscesses and abscess wall samples from patients with HIV infection and AIDS and heightened recognition of abscessing KS underpin the correct diagnosis of this morphological presentation. KS is an AIDS-defining condition and management and staging of patients with AIDS are dependent on its recognition. It is mandatory that the unusual and emerging variants of KS be recognized by clinicians and pathologists to ensure optimal diagnosis and patient management, especially when the KS variant is the sentinel clue to HIV infection and AIDS. The documentation of 'abscessing' KS not only expands the clinicopathological profile of KS but also highlights associated diagnostic mimicry and pathogenetic hiatuses of this enigmatic AIDS-defining malignancy, that require detailed prospective investigation.

\section{Acknowledgement}

Mrs. M Moodley for administrative assistance, Mr. Dinesh Sookhdeo for laboratory support and Mr. S Bandezi, NHLS Management, for research support.

\section{References}

1. Ruocco E, Ruocco V, Tornesello ML, Gambardella A, Wolf R, et al. (2013) Kaposi's sarcoma: Etiology and pathogenesis, inducing factors, causal associations and treatments: Facts and controversies. Clin Dermatol 31: 413422.

2. Radu O, Pantanowitz L (2013) Kaposi sarcoma. Arch Pathol Lab Med 137: 289-294.

3. La Ferla L, Pinzone MR, Nunnari G, Martellotta F, Lleshi A, et al. (2013) Kaposi' s sarcoma in HIV-positive patients: The state of art in the HAART-era. Eur Rev Med Pharmacol Sci 17: 2354-2365.

4. Cattelan AM, Calabro ML, Gasperini P, Aversa SML, Zanchetta M, et al. (2000) Acquired immunodeficiency syndrome-related Kaposi's sarcoma regression after highly active antiretroviral therapy: Biologic correlates of clinical outcome. J Natl Cancer Inst Monogr 28: 44-49.

5. Burdick AE, Carmichael C, Rady PL, Tyring SK, Badiavas E (1997) Resolution of Kaposi's sarcoma associated with undetectable level of human herpesvirus 8 DNA in a patient with AIDS after protease inhibitor therapy. J Am Acad Dermatol 37: 648-649.

6. Busarla SVP, Sayed S, Nazarian RM, Gimbel DC, Moloo Z, et al. (2012) Kaposi sarcoma in association with molluscum contagiosum: An uncommon diagnosis is a single biopsy and potential diagnostic pitfall. Am J Dermatopathol 34: e7-e9.

7. Naidu TK, Ramdial PK, Naidoo SK (2012) Highly active antiretroviral therapyassociated flare of oropharyngeal Kaposi sarcoma. Arch Otolaryngol Head Neck Surg 138: 762-764.

8. Pantanowitz L, Früh K, Marconi S, Moses AV, Dezube BJ (2008) Pathology of rituximab-induced Kaposi sarcoma flare. BMC Clin Pathol 8: 7.

9. Parra R, Leal M, Delgado J, Macias J, Rubio A, et al. (1998) Regression of invasive AIDS-related Kaposi's sarcoma following antiretroviral therapy. Clin Infect Dis 26: 218-219.

10. Ramdial PK, Sing Y, Subrayan S, Calonje E (2010) Cutaneous colesional acquired immunodeficiency syndrome associated Kaposi sarcoma and cryptococcosis. Am J Dermatopathol 32: 780-786.

11. Ramdial PK, Sing Y, Subrayan S, Calonje E, Aboobaker J, et al. (2010) Granulomas in acquired immunodeficiency syndrome-associated cutaneous Kaposi sarcoma: Evidence for a role for Mycobacterium tuberculosis. J Cutan Pathol 37: 827-834.

12. Ramdial PK, Miles E, Pillay B (2016) The emerging histopathologic diagnostic challenges of Kaposi sarcoma in the acquired immunodeficiency syndrome era. Austin J HIVIAIDS Res 3: 1020.

13. Richter E, Schlüter C, Duchrow M, Hahn M, Rüsch-Gerdes S, et al. (1995) An improved method for the species-specific assessment of mycobacteria in routinely formalin-fixed and paraffin-embedded tissues. J Pathol 175: 85-92.

14. Lin CH, Hsu CW, Chiang YJ, Ng KF, Chiu CT (2002) Esophageal and gastric Kaposi's sarcoma presenting as upper gastrointestinal bleeding. Chang Gung Med J 25: 329-332.

15. O'Donnell PJ, Pantanowitz L, Grayson W (2010) Unique histologic variants of cutaneous Kaposi sarcoma. Am J Dermatopathol 32: 244-250.

16. Ramdial PK (2010) Dermatopathological challenges in the human immunodeficiency virus and acquired immunodeficiency era. Histopathology 56: 39-56.

17. Chang Y, Cesarman E, Pessin MS, Lee F, Culpepper J, et al. (1994) Identification of herpes virus-like DNA sequences in AIDS-associated Kaposi's sarcoma. Science 266: 1865-1869. 
18. Cheuk W, Wong KOY, Wong CSC, Dinkel JE, Ben-Dor D, et al. (2004) Immunostaining for Human herpes virus 8 latent nuclear antigen-1 helps distinguish Kaposi sarcoma from its mimickers. Am J Clin Pathol 121: 335-342.

19. Hong A, Davies S, Lee CS (2003) Immunohistochemical detection of the human herpes virus 8 (HHV8) latent nuclear antigen-1 in Kaposi's sarcoma. Pathology 35: 448-450

20. Nuovo M, Nuovo G (2001) Utility of HHV8 RNA detection for differentiating Kaposi's sarcoma from its mimics. J Cutan Pathol 28: 248-255.

21. Patel RM, Goldblum JR, His ED (2004) Immunohistochemical detection of human herpes virus-8 latent nuclear antigen-1 is useful in the diagnosis of Kaposi sarcoma. Mod Pathol 17: 456-460.

22. Ryan P, Aarons S, Murray D, Markham T, O'Sullivan S, et al. (2002) Human herpesvirus 8 (HHV-8) detected in two patients with Kaposi's sarcoma-like pyogenic granuloma. J Clin Pathol 55: 619-622.

23. Urquhart JL, Uzieblo A, Kohler S (2006) Detection of HHV-8 in pyogenic granuloma-like Kaposi sarcoma. Am J Dermatopathol 28: 317-321.

24. MacSween RNM, Whaley K (1992) Inflammation, healing and repair. Muir's textbook of pathology. Edward Arnold, London.

25. Schürch W, Seemayer TA, Gabbiani G (2004) Myofibroblast. Histology for pathologists. Lippincott Williams \& Wilkins, Philadelphia.

26. Sing Y, Ramdial PK (2007) Cryptococcal inflammatory pseudotumors. Am J Surg Pathol 31: 1521-1527.

27. Horsburgh CR Jr (1996) Tuberculosis without tubercles. Tuber Lung Dis 77: 197-198.

28. Nambuya A, Sewankambo N, Mugerwa J, Goodgame R, Lucas S (1988) Tuberculous lymphadenitis associated with human immunodeficiency virus (HIV) in Uganda. J Clin Pathol 41: 93-96.

29. Ramanathan VD, Jawahar MS, Paramasivan CN, Rajaram K, Chandrasekar $\mathrm{K}$, et al. (1999) A histological spectrum of host responses in tuberculous lymphadenitis. Indian J Med Res 109: 212-220.

30. Ridley DS, Ridley MJ (1987) Rationale for the histological spectrum of tuberculosis: A basis for classification. Pathology 19: 186-192.

31. O'Brien JR (1954) Non-reactive tuberculosis. J Clin Pathol 7: 216-225.

32. Bower M, Nelson M, Young AM, Thirlwell C, Newsom-Davis T, et al. (2005) Immune reconstitution inflammatory syndrome associated with Kaposi sarcoma. J Clin Oncol 22: 5224-5228.

33. French MA, Price P, Stone SF (2004) Immune restoration disease after antiretroviral therapy. AIDS 18: 1615-1627

34. Lu CL, Chang SY, Tseng YT, Wu BR, Liu WC, et al. (2013) Immune reconstitution inflammatory syndrome of Kaposi's sarcoma in an HIV-infected patient. J Microbiol Immunol Infect 46: 309-312.

35. Odongo FCA (2013) Fatal disseminated Kaposi's sarcoma due to immune reconstitution inflammatory syndrome following HAART initiation. Case Reports Infect Dis.

36. Uribe-Querol E, Rosales C2 (2015) Neutrophils in cancer: Two sides of the same coin. J Immunol Res 2015: 983698.

37. Burg G, Kempf W, Kazakov DV, Dummer R, Frosch PJ, et al. (2003) Pyogenic lymphoma of the skin: a peculiar variant of primary cutaneous neutrophil-rich CD30+ anaplastic large-cell lymphoma. Clinicopathological study of four cases and review of the literature. $\mathrm{Br} J$ Dermatol 148: 580-586.

38. Mira JA, Fernàndez-Alonso J, Macías J, Sàez C, Japón MA, et al. (2003) Bone involvement and abscess formation by neutrophil-rich CD30+ anaplastic largecell lymphoma mimicking skeletal infection in an AIDS patient. J Infect 47: 7376.

39. Nanda KD, Bhargava D, Sharma B, Dave A (2012) Plasmacytoma masquerading as an abscess. J Investig Clin Dent 3: 236-239.

40. Almefty RO, Tyree TL, Fusco DJ, Coons SW, Nakaji P (2013) Primary histiocytic sarcoma of the brain mimicking cerebral abscess. J Neurosurg Pediatrics 12 : 251-257

41. Hu P, Pang Z, Shen H, Wang G, Sun H, et al. (2015) Tumor-infiltrating neutrophils predict poor outcome in adenocarcinoma of the esophagogastric junction. Tumor Biol 36: 2965-2971.

42. Caruso RA, Belloco R, Pagano M, Bertoli G, Rigoli L, et al. (2002) Prognostic value of intratumoral neutrophils in advanced gastric carcinoma in a high-risk area in Northern Italy. Mod Pathol 15: 831-837.

43. Reid MD, Basturk O, Thirabanjasak D, Hruban RH, Klimstra DS, et al. (2011) Tumor-infiltrating neutrophils in pancreatic neoplasia. Mod Pathol 24: 1612 1619.

44. Zhou S-L, Dai Z, Zhou Z-J, Chen Q, Wang Z, et al. (2014) CXCL5 contributes to tumor metastasis and recurrence of intrahepatic cholangiocarcinoma by recruiting infiltrative intratumoral neutrophils. Carcinogenesis 35: 597-605.

45. Khurana A, Mohapatra PR, Dhingra N (2009) Carcinoma lung masquerading as lung abscess. Clin Respir J 3: 116-117.

46. Benevides L, Morais da Fonseca D, Donate PB, Tiezzi DG, De Carvalho DD, et al. (2015) IL17 promotes mammary tumor progression by changing the behavior of tumor cells and eliciting tumorigenic neutrophils recruitment Cancer Res 75: 3788-3799.

47. Song W, Yeh CR, He D, Wang Y, Xie H, et al. (2015) Infiltratig neutrophils promote renal cell carcinoma progression via VEGFa/HIF2a and estrogen receptor ß signals. Oncotarget 6: 19290-19304.

48. Lin CY, Lin WY, Yeh S, Li L, Chang C (2015) Infiltrating neutrophils increase bladder cancer cell invasion via modulation of androgen receptor (AR)/MMP13 signals. Oncotarget 6: 43081-43089.

49. Yamada Y, Nakagawa T, Sugihara T, Horiuchi T, Yoshizaki U, et al. (2016) Prognostic value of CD66b positive tumor-infiltrating neutrophils in testicular germ cell tumor. BMC Cancer 16: 898.

50. Ventham NT, Hussien MI (2010) Male breast cancer is rare: An initial presentation may be as an abscess. BMJ Case Rep.

51. Barretto J, Wirk B (2012) Early recognition of immune reconstitution inflammatory syndrome leads to avoidance of endotracheal intubation. World J Oncol 3: 194-198.

52. Feldmesser M (2006) Role of neutrophils in invasive aspergillosis. Infect Immun 74: 6514-6516.

53. Haddow LJ, Colebunders R, Meintjies G, Lawn SD, Elliott JH, et al. (2010) Cryptococcal immune reconstitution inflammatory syndrome in HIV-1-infected individuals: Literature review and proposed clinical case definitions. Lancet Infect Dis 10: 791-802.

54. Marais S, Wilkinson KA, Lesosky M, Coussens AK, Deffur A, et al. (2014) Neutrophil-associated central nervous system inflammation in tuberculous meningitis immune reconstitution inflammatory syndrome. Clin Infect Dis 59 : 11638-11647.

55. Murdoch DM, Venter WDF, Van Rie A, Feldman C (2007) Immune reconstitution inflammatory syndrome (IRIS): Review of common infectious manifestations and treatment options. AIDS Res Ther 4: 9.

56. Nelson AM, Yukari C, Lucas SB. (2017) Immune reconstitution inflammatory syndrome (IRIS): What pathologists should know.

57. Cheng C-H, Hsu Y-H (2017) Immune reconstitution inflammatory syndromeassociated disseminated Kaposi's sarcoma in a patient infected with human immunodeficiency virus: Report of an autopsy case. Tzu Chi Med J 29: 41-45.

58. Eng W, Cockerell CJ (2004) Histological features of Kaposi sarcoma in a patient receiving highly active antiviral therapy. Am J Dermatopathol 26: 127-132.

59. Leidner RS, Aboulafia DM (2005) Recrudescent Kaposi's sarcoma after initiation of HAART: A manifestation of immune reconstitution syndrome. AIDS Patient Care STDS19: 635-644.

60. Vicandi B, Jiménez-Heffernan JA, López-Ferrer P, Gamallo C, Viguer JM (1999) Hodgkin's disease mimicking suppurative lymphadenitis: A fine-needle aspiration report of five cases. Diagn Cytopathology 20: 302-306.

61. Laartz BW, Cooper C, Degryse A, Sinnott JT (2005) Wolf in sheep's clothing Advanced Kaposi sarcoma mimicking vulvar abscess. South Med J 98: 475477

62. Butler LM, Jeffery HC, Wheat RL, Rae PC, Townsend K, et al. (2011) Kaposi's sarcoma-associated herpesvirus infection of endothelial cells inhibits neutrophi recruitment through an interleukin-6-dependent mechanism: A new paradigm for viral immune evasion. J Virol 85: 7321-7332.

63. Tomescu C, Law WK, Kedes DH (2003) Surface downregulation of majo histocompatibility complex class I, PE-CAM and ICAM-1 following de novo infection of endothelial cells with Kaposi's sarcoma-associated herpesvirus. Virol 77: 9669-9684. 